\title{
Nutrición e hidratación en el deportista, su impacto en el rendimiento deportivo
}

Nutrition and hydration in the athlete, its impact on sports performance

\author{
Lisett Hernández-Ponce ${ }^{a}$, Mónica S. Carrasco-García ${ }^{b}$, Trinidad L. Fernández-Cortés ${ }^{b}$, \\ Marco A. González-Unzaga ${ }^{b}$, Araceli Ortiz-Polo ${ }^{b}$
}

\begin{abstract}
:
Introduction: The main role of daily diet is to promote sportsmen the fuel energy and the necessary nutrients. During sports, the decrease in physical performance caused by dehydration is frequent, since many athletes do not ingest enough fluids to replace the losses produced. Objective: To describe the nutrition and hydration of the athlete, through a bibliographic analysis, to assess their impact on sports performance. Methodology: In specialized search engines such as Google Scholar, Scielo, Redalyc, Pub Med, Elservier, Academia.edu and Dialnet, a bibliographic review was performed, among which articles, books and web pages were analyzed. Results: Among the factors that determine sports performance, nutrition is one of the most relevant, on the other hand, poor hydration states, not only impact performance, but can also affect the health of the athlete; the effects can produce alterations in physiological homeostasis; There are different markers for evaluating the state of hydration in sport, it is essential to know them and to be able to use them correctly since each one has advantages and disadvantages compared to the rest. Conclusion: A vitally important nutritional strategy is to monitor optimal nutrition and hydration in athletes, with the aim of ensuring effective performance in the execution of sports activities, as well as ensuring that the body performs its functions correctly, achieving skills like strength, endurance, mobility and speed.
\end{abstract}

Keywords:

Nutrition, Hydration, Dehydration, Performance, Hydrating Drinks, Athlete.

Resumen:

Introducción: El principal papel de la alimentación diaria, es promover a los deportistas el combustible energético y los nutrientes necesarios. Durante la práctica deportiva, la disminución de rendimiento físico que se provoca a raíz de la deshidratación, es frecuente, puesto que muchos deportistas no ingieren suficientes fluidos para reponer las pérdidas producidas. Objetivo: Describir la nutrición e hidratación del deportista, mediante un análisis bibliográfico, para valorar su impacto en el rendimiento deportivo. Metodología: En buscadores especializados como Google Académico, Scielo, Redalyc, Pub Med, Elservier, Academia.edu y Dialnet, se realizó una revisión bibliográfica, entre los cuales, se analizaron artículos, libros y páginas web. Resultados: Entre los factores que determinan el rendimiento deportivo, la nutrición es uno de los más relevantes, por otro lado, los estados de hidratación deficientes, no solo impactan en el rendimiento, sino que también pueden afectar la salud del deportista; los efectos pueden producir alteraciones en la homeostasis

\footnotetext{
a Autor de correspondencia: Lisett Hernández Ponce, Universidad Autónoma del Estado de Hidalgo, Instituto de Ciencias de la Salud, https://orcid.org/0000-0001-8429-9422, Email: lizhp97@gmail.com

b Mónica S. Carrasco García, Universidad Autónoma del Estado de Hidalgo, Instituto de Ciencias de la Salud, https://orcid.org/0000-00015513-2842, Email: m.suheily11@gmail.com

b Trinidad L. Fernández-Cortés, Universidad Autónoma del Estado de Hidalgo, Instituto de Ciencias de la Salud, https://orcid.org/0000-00021639-8733, Email: nut_lorenafdz@yahoo.com.mx

b Marco A. González-Unzaga, Universidad Autónoma del Estado de Hidalgo, Instituto de Ciencias de la Salud, https://orcid.org/0000-00024355-8456, Email: marcounzaga@gmail.com

b Araceli Ortiz-Polo, Universidad Autónoma del Estado de Hidalgo, Instituto de Ciencias de la Salud, https://orcid.org/0000-0001-5561-2221, Email: araceli_ortiz4208@uaeh.edu.mx
}

Fecha de recepción: 17/09/2020, Fecha de aceptación: 06/11/2020, Fecha de publicación: 05/06/2021 
fisiológica; existen diferentes marcadores para evaluación del estado de hidratación en el deporte, es imprescindible conocerlos y poder utilizarlos de manera acertada ya que cada uno presenta ventajas e inconvenientes respecto al resto. Conclusión: Una estrategia nutricional de vital importancia es vigilar la óptima nutrición e hidratación en los deportistas, con el objetivo de garantizar un rendimiento eficaz en la ejecución de la actividad deportiva, así como también, asegurar que el organismo realice sus funciones correctamente, consiguiendo habilidades como fuerza, resistencia, movilidad y velocidad.

\section{Palabras Clave:}

Nutrición, Hidratación, Deshidratación, Rendimiento, Bebidas Hidratantes, Deportista.

\section{Introducción}

Durante la práctica deportiva, la disminución de rendimiento físico que se provoca a raíz de la deshidratación, es frecuente, puesto que muchos deportistas no ingieren suficientes fluidos para reponer las pérdidas producidas (Salazar y Barboza, 2016).

Burke (2010) asevera que el principal papel de la alimentación diaria, es promover a los deportistas el combustible energético y los nutrientes necesarios para optimizar las adaptaciones logradas durante los entrenamientos y poder recuperarse rápidamente entre un esfuerzo y otro. Los deportistas también deben alimentarse para permanecer con buena salud, además de, conseguir y mantener una contextura física óptima.

Por lo señalado anteriormente, es de gran importancia escoger una bebida rehidratante apropiada, donde una correcta combinación de macronutrientes y una adecuada selección y cantidad de electrolitos es fundamental, ya que esto permitirá optimizar la rehidratación celular (Castro-Sepúlveda, Astudillo, Mackay y Jorquera, 2016).

Por lo cual, el objetivo de este análisis es describir la nutrición e hidratación del deportista, mediante un análisis bibliográfico, para valorar su impacto en el rendimiento deportivo.

\section{Metodología}

En buscadores especializados como: Google Académico, Scielo, Redalyc, Pub Med, Elservier, Academia.edu y Dialnet, se realizó una revisión bibliográfica, así como también, en revistas especializadas en temas deportivos y Medicina Deportiva, entre los cuales, se analizaron artículos, libros y páginas web. La revisión bibliográfica se realizó en lengua hispana e inglesa.

\section{Resultados}

\section{Rendimiento Deportivo}

El rendimiento deriva de la palabra parformer, adoptada del inglés (1839), que significa cumplir, ejecutar. A su vez, este término viene de parformance, que en francés antiguo significaba cumplimiento. De manera que, podemos definir el rendimiento deportivo como una acción motriz, cuyas reglas fija la institución deportiva, que permite al sujeto expresar sus potencialidades físicas y mentales. Por lo tanto, podemos hablar de rendimiento deportivo, cualquiera que sea el nivel de realización, desde el momento en que la acción optimiza la relación entre las capacidades físicas de una persona y el ejercicio deportivo a realizar (Billat, 2002).

García-Verdugo (2012) refiere que el rendimiento deportivo supone la posibilidad de alcanzar el máximo potencial en las diferentes capacidades durante la competición. Para ello, el deportista deberá desarrollarse mediante un proceso continuo, sistemático y científico que se conoce con el término de "entrenamiento". El rendimiento deportivo depende de una serie de factores o componentes que, de forma interrelacionada hacen que el individuo alcance sus mejores resultados.

Según Cuenca (2008) las cualidades físicas que son base fundamental del rendimiento son cuatro: resistencia, fuerza, velocidad y movilidad. Hohmann, Lames y Letzelter (2005) definen cada uno como:

\section{a) Resistencia}

Capacidad de rendimiento ante el cansancio, es decir, la cualidad física que permite al individuo realizar esfuerzos sin cansarse.

\section{b) Fuerza}

Bajo la perspectiva científica del comportamiento, la capacidad de fuerza puede ser contemplada como la disposición a realizar movimientos con un alto componente de fuerza.

\section{c) Velocidad}

Capacidad de reaccionar y actuar, bajo condiciones libres de cansancio, en el mejor tiempo posible.

d) Movilidad

Se define como la capacidad de poder realizar movimientos con la requerida amplitud de movimiento. Desde el punto de vista funcional y anatómico viene condicionada por la capacidad de estiramiento y por la flexibilidad. Esta última designa el grado de amplitud de 
movimiento de las articulaciones y varía de la estructura corporal de cada persona.

Toda actividad física puede caracterizarse por sus componentes metabólicos dominantes, criterio decisivo para la definición y elaboración de los distintos programas. De esta manera, Barbany (2002) distingue las siguientes modalidades de entrenamiento:

a) Aeróbico, de endurancia o de resistencia aeróbica: basado en ejercicios de carga continua, a baja intensidad y de duración prolongada. Su objetivo es potenciar la aptitud aeróbica y produce afectación general de funciones orgánicas.

b) Anaeróbico, de potencia o de resistencia anaeróbica: con cargas de corta duración y elevada intensidad. Se distinguen dos variantes, desde el punto de vista metabólico (resistencia anaeróbica aláctica y resistencia anaeróbica láctica), como físico (entrenamiento de fuerza y entrenamiento de velocidad). Martínez-Rodríguez (2015) explica que en deportes o actividades físicas de corte más anaeróbico (ANAE), dentro de los que se pueden encontrar por ejemplo las disciplinas de control de peso, normalmente el porcentaje de masa grasa de los competidores es bajo, con un porcentaje alto de masa muscular, que ayuda a los deportistas a mejorar el rendimiento deportivo de cada modalidad.

c) Interválico: con ejercicios de corta duración y relativa intensidad, alternando con fases de reposo ejecutados en períodos de tiempo prolongados. Se solicitan y participan todos los sistemas metabólicos. Para Terrados, CallejaGonzález y Schelling (2011) lo describen como ejercicio mixto aeróbico-anaeróbico, con acciones de alta intensidad intercaladas con momentos de recuperación y pausa.

Algunos ejemplos acuerdo a estas modalidades se visualizan en la Tabla 1.

Tabla 1. Ejemplos de ejercicio físico aeróbico, anaeróbico e interválico.

\begin{tabular}{|c|c|}
\hline Modalidad & Ejemplos \\
\hline $\begin{array}{c}\text { Aeróbico, de potencia o } \\
\text { de resistencia anaeróbica }\end{array}$ & $\begin{array}{c}\text { Caminata, trote, ciclismo, } \\
\text { remo, escalinata, esquí } \\
\text { de fondo simulado, danza } \\
\text { aeróbica, actividad } \\
\text { elíptica. }\end{array}$ \\
\hline $\begin{array}{c}\text { Anaeróbico, de potencia } \\
\text { o de resistencia } \\
\text { anaeróbica }\end{array}$ & $\begin{array}{c}\text { Disciplinas de control de } \\
\text { peso, judo, karate, lucha. }\end{array}$ \\
\hline
\end{tabular}

\begin{tabular}{|c|c|}
\hline $\begin{array}{c}\text { Interválico o mixto } \\
\text { aeróbico-anaeróbico }\end{array}$ & $\begin{array}{c}\text { Deportes de equipo como } \\
\text { el fútbol, hockey al aire } \\
\text { libre o baloncesto y } \\
\text { balonmano en salón. }\end{array}$ \\
\hline
\end{tabular}

Nota. Ejemplos de ejercicio físico con base a sus componentes metabólicos dominantes. Adaptado de "Evaluación De La Aptitud Física Y Prescripción Del Ejercicio", de Heyward, 2008, p. 45; "Efectos de la dieta y práctica de deportes aeróbicos o anaeróbicos sobre los trastornos del comportamiento alimentario", de MartínezRodríguez, 2015, p. 1241 y "Bases fisiológicas comunes para deportes de equipo", de Terrados et al., 2011, p. 86

Barbany (2002) declara que, a pesar de su importancia, el componente metabólico no es el único factor a considerar en la nutrición del deportista, debido a que, en la actividad física y deportiva intervienen otras cualidades mencionadas anteriormente (resistencia, fuerza, etc.), que también deben tenerse en cuenta en la elaboración del programa nutricio, por lo tanto las características del mismo se mencionan en el siguiente apartado.

\section{Nutrición en el deporte}

Entre los factores que determinan el rendimiento deportivo, la nutrición es uno de los más relevantes, además de los factores genéticos del deportista, el tipo de entrenamiento y los factores culturales. Para Olivos, Cuevas, Álvarez y Jorquera (2012) la dieta de los deportistas se centra en tres objetivos principales: aportar la energía apropiada, otorgar nutrientes para la mantención y reparación de los tejidos especialmente del tejido muscular, y mantener y regular el metabolismo corporal.

\section{Hidratos de Carbono (HC)}

Los $\mathrm{HC}$ en el período de entrenamiento, tienen por objetivo la mantención de los depósitos corporales de estos y el aporte adecuado de energía para la ejecución de la actividad física, mediante el aporte de glucosa al músculo esquelético y por el aporte de glucosa y fructosa al hígado, permitiendo la síntesis de glicógeno hepático. A diferencia de una planificación nutricional habitual, la estimación de la cantidad de $\mathrm{HC}$ en la dieta de un deportista no debe ser estimada de acuerdo a las calorías totales de la dieta, sino que idealmente debe ser estimada en relación al peso corporal (Olivos et al., 2012). Así, en función de las horas de entrenamiento diario, los gramos de $\mathrm{HC}$ recomendados se encuentra en la Tabla 2.

Tabla 2. Pautas para la ingesta de hidratos de carbono. 
Nota. Recomendaciones de hidratos de carbono en función de las horas de entrenamiento diario. Adaptado de «La guía completa de la nutrición del deportista», de Bean, 2016.

\section{Proteínas}

Olivos et al. (2012) mencionan que, en el caso de los deportistas, durante la práctica deportiva, las proteínas pueden llegar a aportar entre $5-10 \%$ del total de energía utilizada. La diferencia con alguien que no practica deporte, radica en que posterior al ejercicio se incrementa en forma significativa la síntesis proteica. Los factores determinantes de los requerimientos de proteínas en los deportistas son: el tipo de deporte, la intensidad del ejercicio, la frecuencia del entrenamiento, la ingesta energética a través de la dieta, el contenido de $\mathrm{HC}$ del plan de alimentación y las reservas corporales de HC. Las recomendaciones proteicas se muestran en la Tabla 3.

\begin{tabular}{|l|l|}
\hline \multicolumn{1}{|c|}{ Nivel de actividad } & \multicolumn{1}{|c|}{$\begin{array}{c}\text { Cantidad } \\
\text { recomendable }\end{array}$} \\
\hline $\begin{array}{l}\text { Entrenamiento de fuerza, } \\
\text { etapa de Mantenimiento }\end{array}$ & $\begin{array}{l}1.2-1.4 \mathrm{gr} / \mathrm{kg} \text { de peso } \\
\text { corporal }\end{array}$ \\
\hline $\begin{array}{l}\text { Entrenamiento de fuerza, } \\
\text { etapa de aumento de masa } \\
\text { muscular }\end{array}$ & $\begin{array}{l}1.8-2.0 \mathrm{gr} / \mathrm{kg} \text { de peso } \\
\text { corporal }\end{array}$ \\
\hline $\begin{array}{l}\text { Entrenamiento de resistencia } \\
1.4-1.6 \mathrm{gr} \text { de proteínas } / \mathrm{kg} \\
\text { de peso corporal }\end{array}$ \\
\hline $\begin{array}{l}\text { Actividades intermitentes de } \\
\text { alta Intensidad }\end{array}$ & $\begin{array}{l}1.4-1.7 \mathrm{gr} \text { de proteínas/ } \mathrm{kg} \\
\text { de peso corporal }\end{array}$ \\
\hline Recuperación post-ejercicio & $\begin{array}{l}0.2-0.4 \mathrm{gr} / \mathrm{kg} \text { de peso } \\
\text { corporal }\end{array}$ \\
\hline
\end{tabular}

Tabla 3. Pautas para la ingesta de proteínas

Nota. Recomendaciones de proteínas en función a la etapa de entrenamiento diario. Adaptado de «Nutrición Para el Entrenamiento y la Competición», de Olivos, Cuevas, Álvarez y Jorquera, 2012, p. 256.

Cabe mencionar que, en deportistas entrenados, la ingesta de proteínas en cantidades mayores a lo señalado no otorga beneficios, siendo el exceso de estas oxidado sólo para obtener energía. Bean (2016) explica también que, el excedente se degrada en forma de urea (se excreta).

\section{Lípidos}

Los lípidos son un componente necesario de la dieta, que proporciona energía y elementos esenciales, como las vitaminas A, D, K y E. El rango aceptable es de 20-35\% (el $20 \%$, durante el periodo competitivo, y el $35 \%$, sólo cuando la ingesta de Ácidos Grasos Mono insaturados (AGM) es superior a un 15-20\%) de la ingesta energética total, teniendo en cuenta una proporción del 7-10\% para grasas saturadas (AGS), 10\% para poliinsaturadas (AGP) y superior a $10-15 \%$ de grasas monoinsaturadas (AGM) (Martínez-Sanz, Urdampilleta y Mielgo-Ayuso, 2013).

\begin{tabular}{|l|c|}
\hline \multicolumn{1}{|c|}{ Nivel de actividad } & \multicolumn{1}{c|}{$\begin{array}{c}\text { Cantidad } \\
\text { recomendable }\end{array}$} \\
\hline $\begin{array}{l}\text { Entrenamiento muy ligero } \\
\text { (ejercicio de baja intensidad o } \\
\text { que necesita poca habilidad) }\end{array}$ & $\begin{array}{c}3-5 \mathrm{~g} / \mathrm{kg} \text { de peso } \\
\text { corporal, al día }\end{array}$ \\
\hline $\begin{array}{l}\text { Entrenamiento de intensidad } \\
\text { moderada } \\
\text { (aproximadamente } 1 \mathrm{~h} \text { diaria) }\end{array}$ & $\begin{array}{c}5-7 \mathrm{~g} / \mathrm{kg} \text { de peso } \\
\text { corporal, al día }\end{array}$ \\
\hline $\begin{array}{l}\text { Entrenamiento de intensidad } \\
\text { moderada-alta (1-3h diarias) }\end{array}$ & $\begin{array}{c}7-12 \mathrm{~g} / \mathrm{kg} \text { de peso } \\
\text { corporal, al día }\end{array}$ \\
\hline $\begin{array}{l}\text { Entrenamiento de intensidad } \\
\text { muy alta (>4h diarias) }\end{array}$ & $\begin{array}{c}10-12 \mathrm{~g} / \mathrm{kg} \text { de peso } \\
\text { corporal, al día }\end{array}$ \\
\hline
\end{tabular}

\section{Agua Corporal}

El contenido de agua compone aproximadamente el $60 \%$ del peso corporal; sin embargo, varía a lo largo de la vida en función de distintos factores, tales como la edad, el género, composición muscular, situaciones patológicas, entre otros.

El agua corporal se localiza, en dos compartimentos: como agua intracelular (donde se encuentran $2 / 3$ partes del total), y como agua extracelular (que contiene $1 / 3$ parte de total), repartida entre el espacio intersticial, la sangre, la linfa, las secreciones, etc. (Lizarraga, 2015). En condiciones normales, la entrada de agua en el organismo procede de la ingesta de líquidos (alrededor de $2.300 \mathrm{~mL} /$ día), así como de la producción de agua a través de las reacciones del metabolismo celular (200 mL/día). En cuanto a las fuentes de salida de agua, la principal se produce en forma de orina $(1.500 \mathrm{~mL} /$ día), seguido de la transpiración cutánea (350 mL/día), ventilación pulmonar (350 mL/día), sudor (150 mL/día) y las heces (159 mL/día) (Mielgo-Ayuso et al., 2015).

En el caso de los varones, sobre todo si son deportistas, presentan un componente muscular mayor y, por lo tanto, mayor contenido de agua, puesto que el músculo es un tejido comparativamente más rico en agua que el adiposo (70\% y $10 \%$, respectivamente). Debido a que las pérdidas por sudor aumentan durante el ejercicio, la ingesta de líquidos también debe incrementarse.

El volumen de líquido se renueva constantemente por medio de distintos mecanismos manteniendo la ingesta y la eliminación de agua de forma equilibrada. El balance de agua corporal representa la diferencia entre la ingesta y la pérdida de fluidos, las variaciones de consumo de fluidos son controladas por los riñones, que pueden producir mayor o menor cantidad de orina, dependiendo de los cambios en el volumen de agua corporal (Vega-Pérez, Ruiz-Hurtado, Macías-González, García-Peña, y Torres-Bugarín, 2016).

\section{Hidratación}


Mielgo-Ayuso et al. (2015) indican que el estado de normal de hidratación, a menudo llamado euhidratación, es importante para la salud y el bienestar, es decir, es el estado en el que el individuo sano mantiene el equilibrio hídrico, el cual depende de la diferencia entre las entradas y las pérdidas de agua.

Una buena hidratación es condición fundamental para optimizar el rendimiento deportivo. La importancia de los líquidos, radica en el restablecimiento de la homeostasis del organismo por la pérdida de agua y electrolitos (iones) provocada por la actividad física a través de mecanismos como la sudoración (Vega-Pérez et al., 2016).

Fonseca, Ballesteros y Huesca (2016) enuncian que según el American College of Sports Medicine Position (ACSM por sus siglas en inglés), comenzar la actividad física en un estado de euhidratación y con niveles normales de electrolitos, es uno de los objetivos a cumplir en atletas o personas que se ejercitan. Además, para minimizar o evitar los efectos de la deshidratación, los deportistas se deben asegurar de estar bien hidratados previa y posteriormente del ejercicio, por lo cual, es necesario establecer tácticas de hidratación (Sellés et al., 2015), que se abordarán a continuación.

\section{Pautas de hidratación}

Salazar y Barboza (2016) declaran que es muy importante elaborar una estrategia capaz de mantener un nivel de líquido corporal óptimo mientras se hace ejercicio; así como, en los entrenamientos y en la competición. Dependiendo del tipo de modalidad deportiva, de los factores ambientales, de las características y de la duración de la competición, deberá tenerse en cuenta la realización de un protocolo de hidratación adecuada, utilizando una bebida idónea para cubrir las necesidades hídricas del deportista antes, durante y después del entrenamiento y/o competencia, puesto que se sabe que la hidratación es un factor esencial para mantener la salud en el deportista (Vega-Pérez et al., 2016).

Fonseca et al. (2016) dividen las pautas de hidratación 3 momentos:

\section{a) Antes del ejercicio}

El ACSM recomienda que los atletas se hidraten lentamente antes de hacer ejercicio, en una cantidad de 5 a $7 \mathrm{ml} / \mathrm{kg}$ de peso corporal. Si la orina es muy concentrada o no se ha orinado se recomienda tomar otros 3 a $5 \mathrm{ml} / \mathrm{kg}$ de peso de líquido dos horas antes de hacer ejercicio, para una hidratación adecuada y con el fin de dar suficiente tiempo para que se excrete el exceso de líquido. En días cálidos se aconseja a los atletas que beban entre 250 y $500 \mathrm{ml}$ adicionales entre 30 y 60 minutos antes de hacer ejercicio. Se recomienda que la temperatura de las bebidas se encuentre entre 15 y $21^{\circ} \mathrm{C}$.

\section{b) Durante el ejercicio}

La meta de ingestión de líquido durante la práctica deportiva, es prevenir la deshidratación excesiva ( $>2 \%$ de pérdida de peso corporal por déficit de agua) y los cambios excesivos en el balance de electrolitos; debido a que hay una variabilidad considerable en las tasas de sudoración y el contenido de electrolitos del sudor entre individuos, se recomiendan programas de reposición de líquidos personalizados. Se sugiere que los deportistas ingieran de 400 a $800 \mathrm{ml} / \mathrm{h}$ de líquidos en una distribución de 100 a $200 \mathrm{ml}$ de líquido cada $15 \mathrm{~min}$ durante el ejercicio. Esto después de la primera hora de ejercicio, en donde ya existe una pérdida significativa de líquidos y electrolitos. La tasa de reposición de líquidos depende de la tasa de sudoración del individuo, en la que influye la duración, intensidad, tipo de ejercicio, vestimenta, equipo, condiciones climáticas, predisposición genética, aclimatación al calor, estado de entrenamiento y las oportunidades de beber agua.

\section{c) Después del ejercicio}

La finalidad es reponer completamente cualquier deficiencia de líquidos y electrolitos, en especial el sodio, electrolito que más se pierde en el sudor y el más importante para mantener la osmolalidad. Si el tiempo de recuperación y las oportunidades lo permiten, el consumo de alimentos normales y meriendas con un volumen suficiente de agua natural restaurará la euhidratación. El consumo de sodio durante el periodo de recuperación ayudará a retener los líquidos ingeridos y ayudará a estimular la sed. Por otro lado, se podrán ingerir alimentos especialmente altos en sodio acompañados de agua pura para la recuperación electrolítica del organismo sin la necesidad de ingerir bebidas deportivas. Cuando sea posible, los líquidos deben consumirse espaciados en el tiempo y con suficientes electrolitos porque de lo contrario, el consumo de un mayor volumen de líquido en lugar de hidratar resultará en una mayor producción de orina. Los individuos que buscan alcanzar una recuperación rápida y completa deben beber $1.5 \mathrm{l} / \mathrm{kg}$ de peso corporal perdido, es decir, conviene ingerir de $110-150 \%$ de la pérdida de peso en las primeras seis horas tras el ejercicio para cubrir el líquido eliminado tanto por el sudor como por la orina y de esta manera recuperar el equilibrio hídrico (Vega-Pérez et al., 2016).

\section{Deshidratación}

La deshidratación es la pérdida dinámica de líquido corporal y electrolitos debida al sudor a lo largo de un ejercicio físico sin reposición de líquidos, o en su caso, cuando la reposición no compensa la cantidad perdida (Fonseca et al., 2016).

Los autores anteriores, señalan además que la deshidratación en el deportista es secundaria a diversos factores, entre los que destacan un esfuerzo físico intenso, la restricción de líquidos antes o durante la actividad física, la exposición a un ambiente caluroso y húmedo, el uso de diuréticos o el uso de ropa excesiva ropa durante el ejercicio.

Castro-Sepúlveda et al. (2015) indican que los estados de hidratación deficientes, no solo impactan en el rendimiento deportivo, sino que también pueden afectar la 
salud del deportista; los efectos pueden producir alteraciones en la homeostasis fisiológica, entre los que diversos autores (Vega-Pérez et al., 2016; Mielgo-Ayuso et al., 2015; Marins et al., 2018; Lizarraga, 2015) destacan de manera inicial excesiva sudoración, cefalea intensa, náuseas y sensación de inestabilidad. El aumento de la deshidratación puede manifestarse mediante calambres musculares, apatía, debilidad, desorientación y además afecta la cantidad máxima de oxígeno que el organismo puede absorber, transportar y consumir en determinado tiempo, es decir, el VO2. Si se continúa con el ejercicio, se producirá agotamiento y golpe de calor marcado por el incremento de la temperatura corporal, falta de sudoración e inconsciencia. La pérdida del fluido corporal se ve reflejada en la reducción del volumen plasmático, lo que ocasiona que la presión arterial disminuya y como consecuencia final que haya menor flujo sanguíneo hacia los músculos y la piel. Esta falta de irrigación sanguínea debe ser compensada con el aumento de la frecuencia cardiaca. También se ve afectado el sistema digestivo, lo que provoca desbalance en el vaciado gástrico con la presencia de náuseas, vómito y diarrea, limitando la apetencia de ingerir líquidos. Por lo tanto, la hidratación deficiente puede alterar el balance osmótico intra y extracelular, resultando evidente una disminución tanto del rendimiento físico como de la función cognitiva.

\section{Clasificación de la deshidratación}

Fonseca et al. (2016) clasifican la deshidratación según la cantidad de sales perdidas en relación con la pérdida de agua, tal como se muestra en la Tabla 4:

Tabla 4. Clasificación de la deshidratación

\begin{tabular}{|c|c|}
\hline Clasificación & Características \\
\hline $\begin{array}{c}\text { Deshidratación } \\
\text { isotónica }\end{array}$ & $\begin{array}{l}\text { Las pérdidas de agua y electrolitos } \\
\text { se producen en proporciones } \\
\text { similares, por lo que las células no } \\
\text { se hinchan ni retraen. }\end{array}$ \\
\hline $\begin{array}{c}\text { Deshidratación } \\
\text { hipotónica }\end{array}$ & $\begin{array}{c}\text { Mayor pérdida de electrolitos que } \\
\text { de agua, con una osmolaridad } \\
<280 \mathrm{mmoles} / \mathrm{L} \text { y un nivel sérico } \\
\text { de sodio }<130 \mathrm{mEq} / \mathrm{L} \text {; la célula se } \\
\text { hincha y el espacio extracelular } \\
\text { pierde agua. }\end{array}$ \\
\hline $\begin{array}{l}\text { Deshidratación } \\
\text { hipertónica }\end{array}$ & $\begin{array}{l}\text { La pérdida de agua supera la de } \\
\text { electrolitos, la osmolaridad }>280 \\
\text { mmoles/L y un nivel sérico de sodio } \\
>130 \mathrm{mEq} / \mathrm{L} \text {; el agua pasa del } \\
\text { interior de la célula al exterior y } \\
\text { aumenta el espacio extracelular. }\end{array}$ \\
\hline
\end{tabular}

Nota. Clasificación de la deshidratación en relación de sales perdidas y pérdida de agua «Hidratación en basquetbolistas entre una bebida comercial y una isotónica», de Fonseca et al., 2016, p. 65.

\section{Niveles de deshidratación y sus efectos fisiológicos}

Según Fonseca et al. (2016) el porcentaje de deshidratación (leve, moderada o grave) determina los signos y síntomas que se presentan en el deportista:

a) Deshidratación leve: se presenta una pérdida de peso menor al $5 \%$ del peso corporal, sed intensa, mucosas ligeramente secas (tanto en la mucosa bucal y la conjuntiva del ojo), orina concentrada y oliguria, taquicardia leve y un estado inquieto.

b) Deshidratación moderada: pérdida de 5 al $10 \%$ del peso corporal, está presente el signo de pliegue positivo (al pellizcar el abdomen, la piel se queda y tarda un poco en volver a su sitio), disminuye la eliminación de orina, fontanela mayor hundida, ojos hundidos y ojerosos, hipotensión leve (por disminución del líquido extracelular), somnolencia e hipotermia.

c) Deshidratación grave: el deportista pierde del 10 al 15 $\%$ del peso corporal, las extremidades se presentan frías y cianóticas, hay hipotensión, vasoconstricción periférica, aumenta la taquicardia, mayor oliguria con tendencia a anuria, alteración o pérdida del nivel de conciencia llamativa, más todos los síntomas de leve y moderada.

Los efectos fisiológicos de acuerdo a la pérdida de agua corporal (ACT) determinado en porcentaje se exponen en la Tabla 5.

Tabla 5. Efectos fisiológicos dependiendo del porcentaje de deshidratación

\begin{tabular}{|c|l|}
\hline $\begin{array}{c}\text { Pérdida de } \\
\text { peso }\end{array}$ & \multicolumn{1}{c|}{ Efectos } \\
\hline $1 \%$ & $\begin{array}{l}\text { Umbral de sed. } \\
\text { Incremento del trabajo cardiaco. } \\
\text { Disminución del rendimiento aeróbico en } \\
\text { climas cálidos. }\end{array}$ \\
\hline $2 \%$ & $\begin{array}{l}\text { Sed más intensa, malestar vago, pérdida } \\
\text { de apetito. } \\
\text { Disminución del rendimiento mental y } \\
\text { cognitivo. }\end{array}$ \\
\hline $3 \%$ & $\begin{array}{l}\text { Disminución del volumen sanguíneo. } \\
\text { Aumento del riesgo de contracturas, } \\
\text { calambres y lipotimias. } \\
\text { Reducción del tiempo de reacción, } \\
\text { concentración y discriminación perceptiva. }\end{array}$ \\
\hline $4 \%$ & $\begin{array}{l}\text { Mayor esfuerzo para los trabajos físicos, } \\
\text { náuseas, disminución de la fuerza } \\
\text { muscular. }\end{array}$ \\
\hline
\end{tabular}




\begin{tabular}{|c|c|}
\hline $5 \%$ & $\begin{array}{l}\text { Malestar, fatiga, pérdida de apetito. } \\
\text { Incremento de temperatura corporal hasta } \\
39^{\circ} \mathrm{C} \text {. } \\
\text { Rápida disminución del rendimiento, alto } \\
\text { riesgo de lesiones musculares y } \\
\text { tendinosas. }\end{array}$ \\
\hline $6 \%$ & $\begin{array}{l}\text { Disminución y fallo de los mecanismos de } \\
\text { termorregulación. }\end{array}$ \\
\hline $7 \%$ & Dificultad en la salivación y deglución. \\
\hline$>10 \%$ & $\begin{array}{l}\text { Dificultad de deambulación con } \\
\text { descoordinación y espasticidad. }\end{array}$ \\
\hline $15 \%$ & $\begin{array}{l}\text { Delirio, sequedad de piel, dificultad para } \\
\text { beber agua. }\end{array}$ \\
\hline$>20 \%$ & $\begin{array}{l}\text { La piel se agrieta y sangra. Por encima } \\
\text { estaría la muerte. }\end{array}$ \\
\hline
\end{tabular}

Nota. Síntomas que produce la deshidratación, dependiendo del porcentaje de deshidratación. Adaptado de "Análisis de los patrones de hidratación de gimnastas de élite. Intervención para mejorar el rendimiento", de Fernández, Strunk, Fernández, Jiménez, y Palacios, 2018, p. 290 e «Hidratación en basquetbolistas entre una bebida comercial y una isotónica», de Fonseca et al., 2016, p. 68.

\section{Bebidas hidratantes}

Barbany (2012) sugiere que la rehidratación se debe hacer teniendo en cuenta las diferencias en el vaciamiento gástrico y la absorción intestinal registrada en el ejercicio.

\section{Vaciamiento gástrico}

La absorción de agua y nutrientes se produce esencialmente en el intestino delgado. La absorción de los hidratos de carbono, agua y electrolitos se lleva a cabo en las primeras porciones del intestino delgado (duodeno y yeyuno). Se calcula que las cantidades óptimas de absorción intestinal por hora son de $600-800 \mathrm{ml}$ para el agua y aproximadamente $60 \mathrm{~g}$ para la glucosa (un volumen adecuado sería beber $750 \mathrm{ml}$ de una solución con un $8 \%$ de hidratos de carbono). Cuando se ingieren cantidades superiores a $1 \mathrm{~L}$ de líquido por hora, los excedentes pueden acumularse en el intestino delgado y el colon. Una solución que tarde mucho en evacuarse o que se absorba mal es de poca utilidad para el deportista y puede ser la causa de trastornos gastrointestinales Gil (2010).

\section{Osmolalidad}

Es la medida del número de partículas disueltas en un líquido. La osmolalidad de la bebida determina de qué manera se difundirá el líquido a través de una membrana. Por ejemplo, si se consume una bebida con una osmolalidad relativamente alta, el agua pasa del torrente circulatorio y las células intestinales al intestino. Si se consume una bebida con una osmolalidad relativamente baja, el agua se absorbe del intestino (es decir, la bebida) hacia las células intestinales y el torrente circulatorio
(Bean, 2016). La autora sugiere una clasificación de las bebidas de acuerdo a la osmolaridad que se muestra a continuación.

\section{Clasificación de las bebidas hidratantes}

\section{Según la osmolalidad:}

a) Bebidas hipotónicas: Suele comercializarse como "light" o "agua deportiva". Tienen una osmolalidad relativamente baja, lo que significa que tiene menos partículas (azúcares y electrolitos) por cada $100 \mathrm{ml}$ que los líquidos propios del organismo. Como esta diluida, se absorbe con mayor rapidez que el agua sola. Por lo general, una bebida hipotónica contiene menos de $4 \mathrm{~g}$ de hidratos de carbono por cada $100 \mathrm{ml}$.

b) Bebidas isotónicas: O "bebidas deportivas", tiene la misma osmolalidad que los líquidos del organismo, lo que significa que contiene aproximadamente el mismo número de partículas (azúcares y electrolitos) por $100 \mathrm{ml}$, y por consiguiente es absorbida con tanta rapidez, o más, que el agua. La mayoría de las bebidas isotónicas comerciales contienen entre 4 y $8 \mathrm{~g}$ de hidratos de carbono por $100 \mathrm{ml}$. En teoría, las bebidas isotónicas proporcionan el equilibrio ideal entre la rehidratación y recarga de energías.

c) Bebidas hipertónicas: Como la cola y otros refrescos listos para beber, tienen una osmolalidad más alta que los líquidos del organismo, ya que contienen más partículas (azúcares y electrolitos) por cada $100 \mathrm{ml}$. Esto significa que se absorbe con mayor lentitud que el agua sola. Suelen contener más de $8 \mathrm{~g}$ de hidratos de carbono por cada $100 \mathrm{ml}$.

En cuanto a las bebidas deportivas, Bean (2016) afirma que se dividen en dos categorías principales: bebidas que reemplazan líquidos y bebidas de hidratos de carbono (energéticas).

a) Bebidas que reemplazan líquidos: Son soluciones de electrolitos e hidratos de carbono. Los azúcares que se añaden normalmente son glucosa, sucrosa, fructosa y polímeros de glucosa (maltodextrinas). El objetivo principal de estas bebidas es reemplazar los líquidos con mayor rapidez que el agua, aunque los azúcares extra, también ayudan a mantener los niveles de azúcar en la sangre y ahorran glucógeno. Estas bebidas pueden ser hipotónicas o isotónicas.

b) Bebidas de hidratos de carbono (energéticas): Aportan más hidratos de carbono por $100 \mathrm{ml}$ que las bebidas que reemplazan líquidos. Los hidratos de carbono son aportados sobre todo en forma de maltodextrinas. Su objetivo principal es proporcionar mayores cantidades de hidratos de carbono; por lo general son isotónicas.

En nuestro país, la Procuraduría Federal del Consumidor (PROFECO) (2018) en el mercado de las bebidas hidratantes encontramos tres grupos (Tabla 6):

Tabla 6. Clasificación de bebidas hidratantes según PROFECO 


\begin{tabular}{|c|c|}
\hline $\begin{array}{c}\text { Tipo de } \\
\text { bebida }\end{array}$ & Características \\
\hline $\begin{array}{c}\text { Bebidas para } \\
\text { deportistas }\end{array}$ & $\begin{array}{c}\text { Elaboradas con la disolución de sales } \\
\text { minerales, edulcorantes u otros } \\
\text { ingredientes con el fin de reponer el } \\
\text { agua, energía y electrolitos perdidos } \\
\text { por el cuerpo humano durante el } \\
\text { ejercicio. }\end{array}$ \\
\hline $\begin{array}{c}\text { Bebidas } \\
\text { hidratantes }\end{array}$ & $\begin{array}{c}\text { Este tipo de productos son soluciones } \\
\text { que contienen electrolitos sin } \\
\text { azúcares. }\end{array}$ \\
\hline Electrolitos \\
orales & $\begin{array}{c}\text { De acuerdo con la Ley General de } \\
\text { Salud, en su artículo 226 fracción VI, } \\
\text { son "medicamentos que para } \\
\text { adquirirse no requieren receta médica } \\
\text { y que pueden expenderse en otros } \\
\text { establecimientos que no sean } \\
\text { farmacias", y que en general } \\
\text { contienen glucosa y electrolitos, como } \\
\text { sodio y potasio. }\end{array}$ \\
\hline
\end{tabular}

Nota. Características de los tipos de bebidas hidratantes según PROFECO. Adaptado de "Bebidas Hidratantes", de PROFECO, 2018.

\section{Normativa de las bebidas hidratantes}

La PROFECO enuncia que, tanto a las bebidas hidratantes, como a las bebidas para deportistas, se les aplica la NOM-218-SSA1-2011 Productos y servicios. Bebidas saborizadas no alcohólicas, sus congelados, productos concentrados para prepararlas y bebidas adicionadas con cafeína. Especificaciones y disposiciones sanitarias. Métodos de prueba, en donde se establece que:

Se les llama bebida para deportistas, a las bebidas saborizadas, no alcohólicas, que son elaboradas por la disolución de sales minerales, edulcorantes u otros ingredientes con el fin de reponer el agua, energía y electrolitos perdidos por el cuerpo humano durante el ejercicio. Las bebidas para deportistas deben contener, por lo menos sodio e hidratos de carbono en forma de azúcares, en las siguientes concentraciones: Sodio entre 230 y $575 \mathrm{mg} / \mathrm{L}$ e hidratos de carbono máximo $80 \mathrm{~g} / \mathrm{L}$.

En cuanto al etiquetado nutrimental la norma instituye que las bebidas para deportistas además de la información nutrimental obligatoria, deben incluir los electrolitos y minerales que aporten.

\section{Composición y características de las bebidas hidratantes}

Durante la práctica de ejercicio, pueden producirse pérdidas considerables de líquidos y electrolitos, debido a periodos prolongados de tiempo, condiciones ambientales o climáticas de alta temperatura y concentraciones de humedad relativa bajas. En estas circunstancias, la ingesta inadecuada de líquidos puede conducir a la deshidratación e hiponatremia (Cepero, Padial, Rojas, Romero y De la Cruz, 2016). La ingesta de bebidas con una mezcla de electrolitos ayuda a reponer la pérdida de los mismos producida durante la actividad física, previene la reducción del volumen plasmático, regulan la sensación de sed y retrasan la aparición de fatiga, con el consiguiente beneficio en el rendimiento (Nebot et.al, 2015).

La temperatura ideal de los líquidos según VegaPérez et al. (2016) debe oscilar entre $15-21^{\circ} \mathrm{C}$, ya que, las bebidas más frías vuelven más lenta la absorción, asimismo, en ocasiones pueden provocar lipotimias y desvanecimientos, mientras que las bebidas más calientes no son apetecibles, por lo que se beberá menos cantidad.

Además de las características ya mencionadas, otro factor que se debe tomar en cuenta es el sabor, este debe ser agradable para fomentar la hidratación voluntaria y la rehidratación. En la Tabla 7 se observan las recomendaciones de la composición de las bebidas para tomar durante la práctica deportiva.

\section{Sodio y carbohidratos en las bebidas deportivas}

Gil (2010) indica que los dos hechos demostrados que más contribuyen al desarrollo de fatiga durante el ejercicio físico son la aparición de deshidratación, por la pérdida de agua y electrolitos por el sudor, y la disminución de los hidratos de carbono almacenados en forma de glucógeno en el organismo.

El electrolito que se pierde en mayor cantidad con el sudor, es el sodio (40- $60 \mathrm{mmol} / \mathrm{l})$, es por ello se añade a las bebidas deportivas, con el objetivo de mantener el estímulo de la sed y favorecer la retención renal de líquido.

Tabla 7. Clasificación de bebidas hidratantes según PROFECO

\begin{tabular}{|l|l|}
\hline Aporte & \multicolumn{2}{|c|}{ Nota } \\
\hline Energía & $\begin{array}{l}\text { Desde } 80-350 \text { Kcal/litro (Al } \\
\text { menos el } 75 \% \text { de las calorías } \\
\text { provendrán de hidratos de } \\
\text { carbono con un alto índice } \\
\text { glucémico) }\end{array}$ \\
\hline $\begin{array}{l}|c| \\
\text { carbono }\end{array}$ \\
\hline Sodio & $450-700 \mathrm{mg} / \mathrm{litro}$ \\
\hline Osmolalidad & $200-300 \mathrm{mOsm} / \mathrm{Kg}$ de \\
& agua \\
\hline
\end{tabular}

Nota. Recomendaciones de la composición de las bebidas para la práctica deportiva. Adaptado de «Nutrición para el entrenamiento y la competición", de Olivos, Cuevas, Álvarez y Jorquera, 2012, p. 258. 
Por otra parte, añadir hidratos de carbono en las bebidas permite una absorción más rápida del agua y el sodio, además de retrasar el consumo de glucógeno muscular, por lo que pospone la aparición de fatiga. Mientras que la glucosa, la sacarosa, las maltodextrinas y el almidón no parecen tener diferencias en cuanto al rendimiento, la utilización exclusiva de fructosa puede provocar alteraciones intestinales y entorpecer la absorción de agua. También hay que tener en cuenta que las maltodextrinas tienen un sabor menos dulce que la sacarosa y la fructuosa. Una alta cantidad de HC puede producir cambios digestivos, así como, extraer momentáneamente agua de músculos y piel; por el contrario, una concentración baja de hidratos de carbono puede ser insuficiente.

En resumen, la glucosa estimula la absorción de agua y sodio; a su vez, el sodio es necesario para retransportar la glucosa y optimizar la absorción de fluidos. La glucosa y el sodio, juntos, potencian su mutua absorción y facilitan el trasporte de agua al interior de las células intestinales, al crear un mayor gradiente osmótico (Gil, 2010).

\section{Métodos para valorar la euhidratación}

Existen diferentes marcadores para evaluación del estado de hidratación en el deporte, es de gran importancia conocerlos y poder utilizarlos de manera acertada ya que cada uno presenta ventajas e inconvenientes respecto al resto, incluso, Mielgo-Ayuso et al. (2015) refieren que no existe un acuerdo sobre cuál es el método óptimo para medir el estado de hidratación para aplicarlo universalmente, sin embargo, hay algunos autores que sugieren que el "gold standard" es la combinación de varios de ellos. Algunas técnicas para determinar el estado de hidratación son las siguientes:

\section{Análisis de Impedancia Bioeléctrica (BIA)}

Gil (2010) explica que se trata de un método no invasivo, práctico y fácil de aplicar que permite cuantificar los distintos compartimentos del cuerpo. La impedancia eléctrica es la oposición que presenta un cuerpo (en este caso un tejido biológico) al paso de una corriente a través de él. El procedimiento es realizado mediante la aplicación de una corriente eléctrica alterna de bajo voltaje al cuerpo del individuo, que actúa como conductor; de esta forma, se obtiene el contenido de agua y masa libre de grasa y, por diferencia respecto al peso total corporal, la cantidad de masa grasa (Ramírez, Almanza y Ángel, 2014).

El análisis de la bioimpedancia permite obtener una valoración semicuantitativa del estado de hidratación del paciente en cualquier situación clínica e independientemente del peso corporal (Cubas y Espinoza, 2019).

\section{Osmolalidad plasmática}

Los cambios en el volumen sanguíneo y su composición reflejan cambios en el estado de hidratación. Los análisis de sangre para conocer el estado de hidratación deberán incluir la concentración de hemoglobina y hematocrito, la concentración de sodio y la osmolalidad (Mielgo-Ayuso et al., 2015) (tabla 8). El punto fijo de euhidratación de la osmolalidad del plasma radica en $285 \mathrm{mOsml} / \mathrm{Kg}$ aproximadamente, cuando el atleta se encuentra deshidratado este valor aumenta (Martínez, 2016).

Tabla 8. Indicadores séricos y rangos de referencia de euhidratación

\begin{tabular}{|c|c|}
\hline \multicolumn{2}{|c|}{ Hemoglobina } \\
\hline Hombres & $14,0-17,0 \mathrm{~g} / \mathrm{dL}$ \\
\hline Mujeres & $11,5-16,0 \mathrm{~g} / \mathrm{dL}$ \\
\hline \multicolumn{2}{|c|}{ Hematocrito } \\
\hline Hombres & $42-54 \%$ \\
\hline Mujeres & $38-46 \%$ \\
\hline \multicolumn{2}{|c|}{ Sodio sérico } \\
\hline \multicolumn{2}{|c|}{ 132-142 mmol/L } \\
\hline \multicolumn{2}{|c|}{ Osmolalidad sérica } \\
\hline \multicolumn{2}{|c|}{$280-300$ mOsmol/kg }
\end{tabular}

Nota. Indicadores de hemoglobina, hematocrito, sodio sérico y osmolalidad sérica en hombres y mujeres como referencia de euhidratación. Adaptado de "Valoración del estado nutricional y del gasto energético en deportistas", de Mielgo-Ayuso et al., 2015, p. 233.

\section{Gravedad Específica de Orina (GEO)}

Fonseca et al. (2016) explican que el procedimiento consiste en colocar unas gotas de muestra de orina en la plaquilla de vidrio de un refractómetro hasta esperar los resultados. Cualquier fluido que es más denso que el agua tiene una gravedad específica mayor a 1.000. La orina tiene una gravedad específica que oscila entre 1.013 a 1.029 en adultos sanos. Una distribución más puntual para medir la euhidratación se observa en la Tabla 9.

Tabla 9. Índices de hidratación a partir de la gravedad específica de la orina

\begin{tabular}{|c|c|}
\hline Condición & Gravedad Específica \\
\hline Euhidratación & $<1.020$ \\
\hline Deshidratación mínima & $\geq 1.020$ \\
\hline Deshidratación severa & $>1.030$ \\
\hline
\end{tabular}

Nota. Niveles de euhidratación, deshidratación mínima y deshidratación severa a partir de la gravedad específica de la orina. Adaptado de «Efectos de la deshidratación en atletas de resistencia y estrategias nutricionales para su control», de Martínez, 2016, p. 44. 


\section{Carta de colores de deshidratación de la orina}

La facilidad para valorar el color de la orina hace que, sea cada vez más utilizada por los deportistas, la denominada carta de colores de deshidratación de la orina, en la cual una orina de color amarillo claro, como limonada, corresponde a un adecuado estado de hidratación, mientras que un color oscuro, semejante al zumo de manzana, sería indicativo de deshidratación (Lizarraga, 2015).

Para Mielgo-Ayuso et al. (2015) el color de la orina se determina por la cantidad de urocromo resultante de la degradación de la hemoglobina en la muestra. Las investigaciones han demostrado relaciones lineales entre color de la orina y la gravedad específica, y entre el color de la orina y la conductividad. Por lo tanto, el color de la orina es una forma aceptable para estimar el estado de hidratación en los marcos deportivo o de investigación, los colores más claros indican una hidratación adecuada, mientras que los colores más oscuros indican la necesidad de consumo de líquidos.

\section{Valoración de la masa o peso corporal perdido}

Durante la actividad física se producen cambios en la masa corporal como consecuencia de la pérdida de agua por medio de la respiración y el sudor, lo que puede alterar la homeostasis del volumen intra y extracelular del organismo (Vega-Pérez et al., 2016).

Los cambios el peso corporal, además de cualquier medida de cambio en el agua corporal, se suelen utilizar en los estudios de investigación para cuantificar los cambios en el estado de hidratación (Mielgo-Ayuso et al., 2015). Los mismos, sugieren que las mediciones deben ser realizadas con los sujetos desnudos o con ropa ligera y seca, siendo esta idéntica antes y después de ambas medidas. El test del peso corporal debe ser registrado antes y después del ejercicio. Es conveniente que los sujetos orinen y defequen antes de ser evaluados.

El cálculo del porcentaje de peso perdido se llevó a cabo mediante la siguiente fórmula tomada de CastroSepúlveda et al. (2015):

Porcentaje (\%) Peso Perdido o Deshidratación $=[($ Peso antes - Peso después) / Peso antes] x 100

A partir de estas mediciones, incluyendo otros factores se puede determinar el consumo de líquidos, pérdidas por sudoración y la tasa de sudoración.

\section{Tasa de sudoración}

Marins et al. (2018) expresan que durante la práctica de ejercicio físico se produce un aumento de la temperatura interna; dependiendo de la intensidad del trabajo, de las condiciones ambientales y del tipo de ropa utilizada, se puede aumentar la carga térmica y producirse un importante aumento en la temperatura corporal. Como mecanismo de control de esa temperatura corporal, se produce un aumento en el flujo sanguíneo hacia la piel y, consecuentemente, un incremento en la producción del sudor. La evaporación del sudor es la vía primaria de pérdida de calor que revierte en una continua pérdida de líquidos corporales y electrolitos, principalmente sodio y potasio.

Mielgo-Ayuso et al. (2015) indica que la tasa de sudoración puede calcularse de la siguiente manera:

1. Peso perdido: peso total corporal antes del ejercicio $(\mathrm{kg})$ - peso total corporal después del ejercicio $(\mathrm{kg})$.

2. Sudoración: Peso perdido + cantidad total de líquido ingerido durante el entrenamiento - orina total producida durante el ejercicio $(\mathrm{mL})$.

3. Tasa de sudoración: (sudoración/duración del ejercicio en minutos).

\section{Ayudas Ergogénicas}

En general, se define la ayuda ergogénica como la aplicación de cualquier método o maniobra (ya sea de tipo nutricional, físico, mecánico, psicológico o farmacológico) que se realiza con el fin de mejorar la capacidad de realizar un trabajo físico determinado (Blasco, 2016). El término ergogénesis significa producción de energía. Si una determinada manipulación mejora el rendimiento a través de la producción de energía, se denomina ergogénica y si lo reduce ergolítica (González, Sanchez y Mataix, 2006).

Blasco (2016) puntualiza como ayudas ergogénicas nutricionales a un conjunto de ingestas dirigidas a mantener y/o aumentar el nivel de prestación motora, minimizando las manifestaciones de fatiga sin poner en peligro la salud del deportista.

Bean (2005) expone que el propósito de las ayudas ergogénicas es mejorar el rendimiento físico. Concretamente, se afirma que aumentan el tejido muscular, mejoran la resistencia física, promueven el consumo de grasas y aumentan las fuerzas.

Algunos de los productos ergogénicos más populares se incluyen en la Tabla 10.

Tabla 10. Indicadores séricos y rangos de referencia de euhidratación

\begin{tabular}{|l|}
\hline \multicolumn{2}{|c|}{ Ayudas ergogénicas } \\
\hline - Suplementos antioxidantes \\
- Suolementos proteicos \\
- Suplementos de aminoácidos de \\
- cadena ramificada (AACR) \\
- Croductos sustitutirios de comidas \\
- Glutamina \\
- Ácido Beta-Hidroxi Beta-Metilbutírico \\
- (HMB) \\
- Pehidroepiandrosterona (DHEA) \\
\hline
\end{tabular}




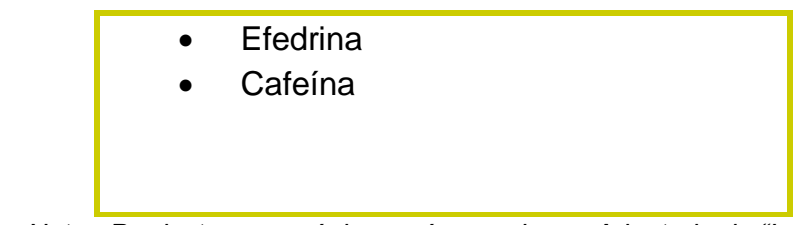

Nota. Productos ergogénicos más populares. Adaptado de "La guía completa de la nutrición del deportista", de Bean, 2005, p. 91-92.

\section{Conclusión}

El cuerpo humano, en específico sus sistemas fisiológicos no podrían ser posibles sin los niveles de hidratación adecuados, por lo que la más mínima variación puede provocar efectos negativos en la salud del individuo. Un deportista no es la excepción, pese a las adaptaciones corporales que presenta, un estado de deshidratación, no solo va a provocar una disminución en el rendimiento físico, sino que además aumenta el riesgo de lesiones, y puede poner en juego la salud e incluso la vida del atleta, es por ello que, se deben tomar en cuenta los diferentes métodos para evaluar el estado de hidratación del deportista, donde el papel del nutriólogo es fundamental, puesto que es el profesional responsable de acompañar y orientar al atleta.

Una estrategia nutricional de vital importancia es vigilar la óptima nutrición e hidratación en los deportistas, con el objetivo de garantizar un rendimiento eficaz en la ejecución de la actividad deportiva, así como también, asegurar que el organismo realice sus funciones correctamente, consiguiendo habilidades como fuerza, resistencia, movilidad y velocidad.

\section{Referencias}

[1] Barbany, J.R. (2002). Fisiología del ejercicio físico y del entrenamiento. Recuperado de:

https://books.google.es/books?hl=es\&lr=\&id=kq0XqZoY8YoC\&oi=f nd\&pg=PA5\&dq=clasificacion+de+ejercicio+fisico+en+base+a+meta bolismo+energ\%C3\%A9tico\&ots=DovygLvrHB\&sig=Y5PvckLFPrp pTF6fQJVzHdJ_Y3M\#v=onepage\&q=clasificaci\%C3\%B3n\%20del\% 20ejercicio\&f=false

[2] Barbany, J. R. (2012). Alimentación para el deporte y la salud (1 ${ }^{\mathrm{a}}$ ed.). Barcelona: Paidotribo.

[3] Bean, A. (2005). La guía completa de la nutrición del deportista. Recuperado de:

https://books.google.com.mx/books?id=8ez9YipinNQC\&pg=PA91\& $\mathrm{dq}=$ ayudas+ergogenicas\&hl=es419\&sa=X\&ved=2ahUKEwjRlO2umt bsAhUIRqwKHRvRBYwQ6AEwBHoECAMQAg\#v=onepage\&q=ay udas\%20ergogenicas $\& \mathrm{f}=$ false

[4] Bean, A. (2016). La guía completa de la nutrición del deportista (5 ed.). Barcelona: Paidotribo.

[5] Billat, V. (2002). Fisiología y metodología del entrenamiento: De la teoría a la práctica. Recuperado de https://www.academia.edu/10943974/FISIOLOG\%C3\%8DA_Y_ME TODOLOG\%C3\%8DA DEL ENTRENAMIENTO
[6] Blasco, R. (2016). Las ayudas ergogénicas nutricionales en el ámbito deportivo. Primera parte. Aspectos generales. Nutrición Clínica en Medicina, X (2), 69-78. doi: 10.7400/NCM.2016.10.2.5038

[7] Burke, L. (2010). Nutrición en el deporte: un enfoque práctico ( $6^{\mathrm{a}}$ ed.). Madrid: Médica panamericana.

[8] Castro-Sepúlveda, M., Astudillo, S., Álvarez, C., Zapata-Lamana, R., Zbinden-Foncea, H., Ramírez-Campillo, R. y Jorquera, C. (2015) Prevalencia de deshidratación en futbolistas profesionales chilenos antes del entrenamiento. Nutrición Hospitalaria, 32(1), 308-311. doi: 10.3305/nh.2015.32.1.8881

[9] Castro-Sepúlveda, M., Astudillo, S., Mackay, K. y Jorquera, C. (2016) El consumo de leche posterior al ejercicio disminuye la excreción de electrolitos. Revista Internacional de Medicina y Ciencias de la Actividad Física y el Deporte, 16(62), 221-228. doi: http://dx.doi.org/10.15366/rimcafd2016.62.003

[10] Cepero, M., Padial, R., Rojas, F. J., Romero, D. y De la Cruz, J. C. (2016). Efectos de bebidas carbohidratadas y proteicas sobre la recuperación del esfuerzo. Revista Internacional de Medicina y Ciencias de la Actividad Física y el Deporte, 16(62), 373-401. Recuperado de

Http://cdeporte.rediris.es/revista/revista62/artefectos701.htm

[11] Cubas, C. y Espinoza, M. (2019). Evaluación del estado nutricional mediante bioimpedancia inbody S10 en pacientes hospitalizados con insuficiencia renal crónica terminal en hemodiálisis del hospital Guillermo Almenara Irigoyen. (Tesis de Fin de Grado. Universidad Nacional José Faustino Sánchez Carrión, Perú). Recuperado de http://repositorio.unjfsc.edu.pe/bitstream/handle/UNJFSC/3406/TESI S\%20CUBAS\%20Y\%20ESPINOZA.pdf?sequence $=1 \&$ isAllowed $=y$

[12] Cuenca, F. (2008). Mejora tu rendimiento: Medicina deportiva aplicada al fútbol base. Recuperado de https://books.google.com.mx/books?id=YufEXkHHMrwC\&pg=PA57 $\& \mathrm{dq}=$ cualidades $+\mathrm{f} \% \mathrm{C} 3 \%$ ADsicas $+q u e+$ son+base+fundamental+del+ rendimiento+son+cuatro:+resistencia,+fuerza,+velocidad+y+cuenca\& $\mathrm{hl}=\mathrm{es} \& \mathrm{sa}=\mathrm{X} \& \mathrm{ved}=0$ ahUKEwjA8ry7ve3nAhUPnKwKHfpJAWEQ6A EIKDAA\#v=onepage $\& q=$ cualidades $\% 20 \mathrm{f} \% \mathrm{C} 3 \%$ ADsicas $\% 20$ que $\% 2$ 0son $\% 20 \mathrm{base} \% 20$ fundamental $\% 20 \mathrm{del} \% 20$ rendimiento $\% 20$ son $\% 20 \mathrm{cu}$ atro $\% 3 \mathrm{~A} \% 20$ resistencia $\% 2 \mathrm{C} \% 20$ fuerza $\% 2 \mathrm{C} \% 20$ velocidad $\% 20 \mathrm{y} \% 20$ cuenca\&f=false

[13] Fernández, J., Strunk, R., Fernández, J., Jiménez, S. y Palacios,N (2018). Análisis de los patrones de hidratación de gimnastas de élite. Intervención para mejorar el rendimiento. Archivos de Medicina del Deporte, 35(5), 289-297. Recuperado de http://archivosdemedicinadeldeporte.com/articulos/upload/Femede 1 $\underline{87 \text { web.pdf\#page }=9}$

[14] Fonseca, R. E., Ballesteros, J. C. y Huesca, J. M. (2016). Hidratación en basquetbolistas entre una bebida comercial y una isotónica. Revista Mexicana de Investigación en Cultura Física y Deporte, 9(5), 64-78. Recuperado de http://www.ened.gob.mx/Documentos/REVISTA\%20ENED/Revista9 /articulo5.pdf

[15] García-Verdugo, M. (2012). Resistencia y entrenamiento: Una metodología práctica. Recuperado de https://books.google.com.mx/books?id=zViRDwAAQBAJ\&amp;pg= PT129\&amp;lpg=PT129\&amp;dq=La\%2Bresistencia\%2C\%2Bigual $\% 2 \mathrm{Bque} \% 2 \mathrm{~B}$ otras $\% 2 \mathrm{~B}$ capacidades $\% 2 \mathrm{Bmotrices} \% 2 \mathrm{C} \% 2 \mathrm{Bpuede} \% 2 \mathrm{~B}$ considerarse $\% 2 \mathrm{Buna} \% 2 \mathrm{Bmanifestaci} \% \mathrm{C} 3 \% \mathrm{~B} 3 \mathrm{n} \% 2 \mathrm{Bde} \% 2 \mathrm{Bla} \% 2 \mathrm{Bco}$ ntracci\%C3\%B3n\%2Bmuscular\%2C $\% 2 \mathrm{Bes} \% 2 \mathrm{Bdecir} \% 2 \mathrm{C} \% 2 \mathrm{Bde} \% 2$ Bla\%2Bfundamental $\% 2 \mathrm{Bfunci} \% \mathrm{C} 3 \% \mathrm{~B} 3 \mathrm{n} \% 2 \mathrm{Bpor} \% 2 \mathrm{Bla} \% 2 \mathrm{Bque} \% 2$ Bel\%2Bm\%C3\%BAsculo\%2Bes\%2Bllamado\%2Ba\%2Bdesarrollar\% 2Bmovimiento $\% 2 \mathrm{Bo} \% 2 \mathrm{Btensi} \% \mathrm{C} 3 \% \mathrm{~B} 3 \mathrm{n} . \% 2 \mathrm{BEl} \% 2 \mathrm{Bm} \% \mathrm{C} 3 \% \mathrm{BAsc}$ ulo $\% 2 \mathrm{C} \% 2 \mathrm{Bcuando} \% 2 \mathrm{Bse} \% 2 \mathrm{Bcontrae} \% 2 \mathrm{C} \% 2 \mathrm{Blo} \% 2 \mathrm{Bhace} \% 2 \mathrm{Bcon}$ 
$\% 2 \mathrm{Buna} \% 2 \mathrm{~B}$ fuerza $\% 2 \mathrm{By} \% 2 \mathrm{Buna} \% 2 \mathrm{Bvelocidad} \% 2 \mathrm{Bdeterminadas} \%$ 2BPor\%2Blo\%2Btanto\%2C\%2Bson\%2Bestas\%2Bcaracter\%C3\%AD sticas\%2Blas\%2Bcondicionantes\%2Bdel\%2Btipo\%2Bde\%2Besfuerz o\%2Bque\%2Brealiza.\&amp;source=bl\&amp;ots=suqvPMsQ74\&amp ;sig=ACfU3U0pYwlMbA Uq6fUjonLh6XXfc0g2g\&amp;hl=es\&am p;sa=X\&amp;ved=2ahUKEwj9gu7X8oPnAhXRAp0JHQX6Cb0Q6A EwAHoECAoQAQ\%23v\%3Donepage\&amp;q\&amp;f=false

[16] Gil, A. (2010). Tratado de nutrición: Nutrición clínica (2a ed.). Madrid: Médica Panamericana.

[17] González, J., Sanchez, P. y Mataix, J. (2006). Nutrición en el deporte: ayudas ergogénicas y dopaje. Recuperado de: https://books.google.com.mx/books?hl=es\&lr=\&id=ZBip31e QacC\& oi=fnd\&pg=PA181\&dq=ayudas+ergogenicas\&ots=hb2 mC6N7UC\&s

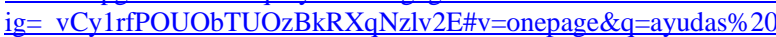
ergogenicas\&f=false

[18] Heyward, V. (2008). Evaluación De La Aptitud Física Y Prescripción Del Ejercicio. Recuperado de:

https://books.google.com.mx/books?id=zn3dDE0R3IMC\&pg=PA45 $\& \mathrm{dq}=$ ejercicio+aerobico\&hl=es-

419\&sa $=$ X\&ved $=2$ ahUKEwjI4sWX0dXsAhVHRqwKHe1vBcYQ6A EwAHoECAEQAg\#v=onepage \&q=ejercicio\%20anaerobico\&f $=$ false

[19] Hohmann, A., Lames, M., Letzelter, M. (2005). Introducción a la ciencia del entrenamiento (1. ${ }^{a}$ ed.). España: Editorial Paidotribo.

[20] Lizarraga, M. A. (2015). Tercera ponència: Proteïnes, fibra dietètica. Hidratació i Salut: Hidratación en diversas edades y actividades deportivas. Revista de la Reial Acadèmia de Medicina de Catalunya, 30(3), 126-129. Recuperado de https://www.raco.cat/index.php/RevistaRAMC/search/results?query=1 izarraga\&searchField $=$

[21] Marins, J. C., Pereira, L., Amorim, P. R., Arnaiz-Lastras, J., SilleroQuintana, M. y Alfenas, C. R. (2018). Suplementos de carbohidratos durante un ejercicio: efectos sobre los electrolitos y glucosa. Revista Internacional de Medicina y Ciencias de la Actividad Física y el Deporte, 18(70), 269-287. doi: http://dx.doi.org/10.15366/rimcafd2018.70.005

[22] Martínez, H. J. (2016). Efectos de la deshidratación en atletas de resistencia y estrategias nutricionales para su control. (Tesis Profesional. Universidad de Sonora, México). Recuperado de http://www.repositorioinstitucional.uson.mx/bitstream/handle/unison/ 1306/martinezmartinezhumbertojordanl.pdf?sequence $=1 \&$ isAllowed $=$ y

[23] Martínez-Rodríguez, A. (2015). Efectos de la dieta y práctica de deportes aeróbicos o anaeróbicos sobre los trastornos del comportamiento alimentario. Nutrición Hospitalaria, 31 (3), 12401245. doi:10.3305/nh.2015.31.3.8131

[24] Martínez-Sanz, J.M., Urdampilleta, A. y Mielgo-Ayuso, J. (2013). Necesidades energéticas, hídricas y nutricionales en el deporte. Motricidad. European Journal of Human Movement, 30, 37-52. Recuperado de https://www.redalyc.org/pdf/2742/274228060004.pdf

[25] Mielgo-Ayuso, J., Maroto-Sánchez, B., Luzardo-Socorro, R., Palacios, G., Palacios, N. y González-Gross, M. (2015). Valoración del estado nutricional y del gasto energético en deportistas. Revista Española de Nutrición clínica y dietética hospitalaria, 21(Supl.1), 225-234. doi: 10.14642/RENC.2015.21.sup1.5069

[26] Nebot, V., Drehmer, E., Elvira, L., Sales, S., Sanchís, C., Esquius, L. y Pablos, A. (2015). Efectos de la ingesta voluntaria de líquidos (agua y bebida deportiva) en corredores por montaña amateurs. Nutrición Hospitalaria, 32(5), 2198-2207. doi:10.3305/nh.2015.32.5.9637
[27] NOM-218-SSA1-2011, Productos y servicios. Bebidas saborizadas no alcohólicas, sus congelados, productos concentrados para prepararlas y bebidas adicionadas con cafeína. Especificaciones y disposiciones sanitarias. Métodos de prueba. Recuperado de http://www.salud.gob.mx/cdi/nom/compi/NOM-218-SSA1-2011.pdf

[28] Procuraduría Federal del Consumidor (2018).Bebidas hidratantes. Revista del Consumidor, (495), 30-39. Recuperado de https://issuu.com/profeco/docs/revista_del_consumidor_mayo_2018/3

[29] Ramírez, D., Almanza, D. y Ángel, L. (2014). Estimación del agua corporal total y del peso seco, usando impedancia bioeléctrica tetrapolar de multifrecuencia (BIA4) en pacientes en hemodiálisis. Revista de la Facultad de Medicina, 63(1), 19-31. doi: https://doi.org/10.15446/revfacmed.v63n1.41183

[30] Salazar, J. P. y Barboza, J. N. (2016). Valores de Sodio y Potasio en suero y su impacto en variación pre y pos ejercicio de actividades fuertes en deportistas. Revista de Ciencias Biológicas y de la Salud, 18(2), 24-26. http://dx.doi.org/10.18633/bt.v18i2.276

[31] Sellés, M. C., Martínez-Sanz, J. M., Mielgo-Ayuso, J., Selles, S., Norte-Navarro, A., Ortiz-Moncada, R. y Cejuela, R. (2015). Evaluación de la ingesta de líquido, pérdida de peso y tasa de sudoración en jóvenes triatletas. Revista Española de Nutrición Humana y Dietética, 19(3), 132-139. doi: http://dx.doi.org/10.14306/renhyd.19.3.146

[32] Terrados, N., Calleja-González, J. y Schelling, X. (2011). Bases fisiológicas comunes para deportes de equipo. Revista Andaluza de Medicina del Deporte, 4(2), 84-88. Recuperado de: https://www.redalyc.org/articulo.oa?id=3233/323327666007

[33] Vega-Pérez, R., Ruiz-Hurtado, K. E., Macías-González, J., GarcíaPeña, M. D. y Torres-Bugarín, O. (2016). Impacto de la nutrición e hidratación en el deporte. El Residente, 11(2): 81-87. Recuperado de https://www.medigraphic.com/pdfs/residente/rr-2016/rr162d.pdf 